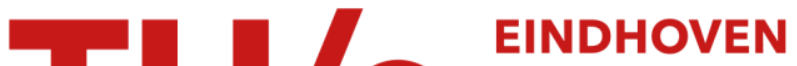 UNIVERSITY OF TECHNOLOGY
}

\section{Individual differences in the rubber-hand illusion : predicting self-reports of people's personal experiences}

\section{Citation for published version (APA):}

Haans, A., Kaiser, F. G., Bouwhuis, D. G., \& IJsselsteijn, W. A. (2012). Individual differences in the rubber-hand illusion : predicting self-reports of people's personal experiences. Acta psychologica, 141(2), 169-177. https://doi.org/10.1016/j.actpsy.2012.07.016

DOI:

10.1016/j.actpsy.2012.07.016

Document status and date:

Published: 01/01/2012

\section{Document Version:}

Publisher's PDF, also known as Version of Record (includes final page, issue and volume numbers)

\section{Please check the document version of this publication:}

- A submitted manuscript is the version of the article upon submission and before peer-review. There can be important differences between the submitted version and the official published version of record. People interested in the research are advised to contact the author for the final version of the publication, or visit the $\mathrm{DOI}$ to the publisher's website.

- The final author version and the galley proof are versions of the publication after peer review.

- The final published version features the final layout of the paper including the volume, issue and page numbers.

Link to publication

\section{General rights}

Copyright and moral rights for the publications made accessible in the public portal are retained by the authors and/or other copyright owners and it is a condition of accessing publications that users recognise and abide by the legal requirements associated with these rights.

- Users may download and print one copy of any publication from the public portal for the purpose of private study or research.

- You may not further distribute the material or use it for any profit-making activity or commercial gain

- You may freely distribute the URL identifying the publication in the public portal.

If the publication is distributed under the terms of Article 25fa of the Dutch Copyright Act, indicated by the "Taverne" license above, please follow below link for the End User Agreement:

www.tue.nl/taverne

Take down policy

If you believe that this document breaches copyright please contact us at:

openaccess@tue.nl

providing details and we will investigate your claim. 


\title{
Individual differences in the rubber-hand illusion: Predicting self-reports of people's personal experiences
}

\author{
Antal Haans ${ }^{\text {a,* }}$, Florian G. Kaiser ${ }^{b}$, Don G. Bouwhuis ${ }^{a}$, Wijnand A. IJsselsteijn ${ }^{\text {a }}$ \\ a Eindhoven University of Technology, The Netherlands \\ ${ }^{\mathrm{b}}$ Otto-von-Guericke University, Magdeburg, Germany
}

\section{A R T I C L E I N F O}

Article history:

Received 29 November 2011

Received in revised form 6 July 2012

Accepted 31 July 2012

Available online $\mathrm{xxxx}$

\section{PsychINFO classification:}

2320 Sensory Perception

2220 Tests \& Testing

Keywords:

Illusions (perception)

Body image disturbances

Individual differences

Rubber-hand illusion

Measurement

\begin{abstract}
A B S T R A C T
Can we assess individual differences in the extent to which a person perceives the rubber-hand illusion on the basis of self-reported experiences? In this research, we develop such an instrument using Rasch-type models. In our conception, incorporating an object (e.g., a rubber hand) into one's body image requires various sensorimotor and cognitive processes. The extent to which people can meet these requirements thus determines how intensely people experience and, simultaneously, describe the illusion. As a consequence, individual differences in people's susceptibility to the rubber-hand illusion can be determined by inspecting reports of their personal experiences. The proposed model turned out to be functional in its capability to predict self-reports of people's experiences and to reliably assess individual differences in susceptibility to the illusion. Regarding validity, we found a small, but significant, correlation between individual susceptibility and proprioceptive drift. Additionally, we found that asynchrony, and tapping rather than stroking the fingers constrain the experience of the illusion.
\end{abstract}

(c) 2012 Elsevier B.V. All rights reserved.

\section{Introduction}

By simultaneously stroking a person's concealed hand together with a visible fake one, people start to sense the fake hand as an actual part of their own body (Botvinick \& Cohen, 1998). In this so-called rubberhand illusion, the central nervous system categorizes a foreign object, for example a rubber hand, as a part of the body. The cognitive and sensorimotor mechanisms underlying such body image incorporations include visuotactile integration (e.g., Armel \& Ramachandran, 2003), and the detection of body-specific sensorimotor contingencies (e.g., Botvinick, 2004). At the same time, the strength of illusion appears to be modulated by an internal model of what the human body is like: Objects that differ morphologically from the human body are less easily incorporated (e.g., de Vignemont, Tsakiris, \& Haggard, 2006; Tsakiris \& Haggard, 2005). The rubber-hand illusion has been used extensively as an experimental paradigm in research aimed at understanding the mechanisms behind our sense of embodiment (e.g., Longo, Schüür, Kammers, Tsakiris, \& Haggard, 2008), personal impressions of body-ownership and self-consciousness (e.g., Ehrsson, Spence, \& Passingham, 2004; Lenggenhager, Tadi, Metzinger, \& Blanke, 2007), or self-other merging (e.g., Paladino, Mazzurega, Pavani, \& Schubert, 2010).

\footnotetext{
* Corresponding author at: Eindhoven University of Technology (IPO 1.35), P.O. Box 513, NL-5600 MB Eindhoven, The Netherlands. Tel.: + 31402475237.

E-mail address: A.Haans@tue.nl (A. Haans).
}

To assess the extent to which the fake hand is incorporated in the body image, research has relied heavily on the measurement of proprioceptive drift (i.e., the degree to which people misperceive the location of their concealed hand as being shifted toward the fake hand; e.g., Tsakiris \& Haggard, 2005). Empirical evidence, however, suggests that this drift in proprioception might not be a valid indicator of the rubber-hand illusion (e.g., Holmes, Snijders, \& Spence, 2006; Folegatti, Farnè, Salemme, \& de Vignemont, 2012; but see Longo et al., 2008). Rohde, Di Luca, and Ernst (2011) have even argued that the rubber-hand illusion is perhaps better assessed using people's descriptions of what the rubber-hand illusion feels like. These descriptions, however, vary widely between individuals. Some people claim, for example, that they felt as if they could move and use the fake hand (i.e., a sense of agency; Haans, IJsselsteijn, \& de Kort, 2008). Other people, under similar experimental conditions, claim that they even saw the fake hand changing appearance (i.e., a case of altered visual perceptions; Lewis \& Lloyd, 2010). Still others appear to be relatively insensitive to the illusion, as the descriptions of their experiences are limited to impressions of strangeness and confusion. The question is: Is this apparent variability in reports about the rubber-hand illusion largely irrelevant? Should it be regarded as noise in the data? Or, alternatively, are these reports of various personal experiences valid indicators of people's differential susceptibility to the illusion?

In this paper, we present two experiments in which we test a model that predicts self-reports of how a person experiences the rubber-hand 
illusion. In a first experiment, we investigate whether individual differences in the susceptibility to the rubber-hand illusion can be used to determine how people describe their personal experiences. In a second experiment, we extend our model to include the effects of experimental manipulations of the situation in which the illusion is elicited (e.g., the amount of asynchrony in the visuotactile stimulation). Before doing so, we discuss research employing Botvinick and Cohen (1998), which is thought to capture experiences related to the rubber-hand illusion.

\subsection{Self-reported experiences}

The measure by Botvinick and Cohen (1998) consists of nine statements regarding specific experiential effects presumed to be related to the rubber-hand illusion. If we compare the various studies that have used the instrument, then it becomes apparent that there is a consistent order with respect to how frequent these various statements are agreed upon (e.g., Ehrsson et al., 2004; Peled, Ritsner, Hirschmann, Geva, \& Modai, 2000; see also Holmes \& Spence, 2007). The three most frequently endorsed statements relate to the location of felt touch (e.g., feeling the touch in the location where one sees the rubber hand being touched), and to a sense of ownership toward the fake hand (e.g., the fake hand feels as one's own). The remaining six statements are much less frequently endorsed (e.g., relating to distortions in the perceived appearance of the fake hand). For most people, these latter statements apparently do not match their experiences.

Some authors have argued that the rarely supported items refer to experiential effects that are unrelated to the illusion (e.g., Ehrsson et al., 2004). Consider, however, two items in a mathematical test: an addition and an integration task. Fewer people will correctly solve the integration than the addition task. This is not because integration is less of a mathematical problem than addition. Instead, integration problems require rather more mathematical skills than addition problems in order to be solved correctly. In other words, the addition and the integration item both need to be matched with a certain level of mathematical ability. We propose an analogous look at the items of Botvinick and Cohen (1998) instrument. The least frequently acknowledged experiential effects are the ones that, in order to be experienced, have to be matched with a high level of a specific ability. We call this ability "susceptibility to the rubber-hand illusion."

\subsection{Individual susceptibility as an ability}

Individual differences in people's susceptibility to the rubber-hand illusion are rather likely, not the least, because individual differences are common with other bodily illusions as well (e.g., MacLachlan, Desmond, \& Horgan, 2003; Mussap \& Salton, 2006). These differences might derive from differential information processing capabilities (e.g., with respect to visuotactile integration; Peled et al., 2000), or from differences in people's psychological makeup (Juhel \& Neiger, 1993). People may, for example, differ in the stability of their body image (Mussap \& Salton, 2006). Burrack and Brugger (2005) found the frequency of spontaneous body image alterations in everyday life to be correlated with people's susceptibility to experimentally induced bodily illusions. The less stable one's body image is, the more likely it is to be sensitive to change due to novel sensorimotor information.

Susceptibility to the rubber-hand illusion reflects the extent to which a person can activate the required sensorimotor and cognitive processes (e.g., visuotactile integration), and inhibit others (e.g., comparing the foreign object with an internal model of the human body). Vice versa, we expect that each experiential effect related to the rubber-hand illusion (e.g., a sense of ownership over the fake hand) demands certain cognitive and sensorimotor processes to be activated or inhibited. We call these experience-specific requirements "cognitive demand". If a person's susceptibility does not match the cognitive demand behind a particular experiential effect, then he or she will not develop, and thus not report, that experience.

\subsection{A transitive order of experiential effects}

Cognitive demand is defined as a property of an experiential effect (e.g., fake hand feels as one's own), and is assumed independent of the individual on whom the rubber-hand illusion is induced. A more susceptible person has better chances of developing and thus reporting a particular experiential effect compared to a less susceptible individual, but the cognitive demand behind that experience is the same for both. In other words, we expect the cognitive demand behind each experiential effect to be more or less the same for everyone. This rests on the assumption that the cognitive and sensorimotor requirements behind body image incorporations are more or less the same for each individual. Note that a similar assumption is made in cognitive psychology, where it is assumed that the cognitive processes behind mental capacities are equivalent for healthy adults.

If this invariance of cognitive demands assumption holds, then all experiential effects related to the rubber-hand illusion can be transitively ordered according to their cognitive demand in a manner that is the same for everyone. An order of objects is called transitive under the conditions exemplified by the following: If experience $A$ is more demanding to develop than experience $\mathrm{B}$, and $\mathrm{B}$ is more demanding than experience $C$, then $A$ must be more demanding than C. This transitive and invariant order of experiential effects implies that a person's susceptibility to the rubber-hand illusion manifests itself in a determined, and thus predictable, set of reported experiences (i.e., all but the too demanding ones).

For people who are marginally susceptible to the rubber-hand illusion, the illusion presumably is limited to impressions of confusion and strangeness as such experiential effects only require registering the contradiction in the sensory information (cf. Armel \& Ramachandran, 2003). Such unreceptive individuals will not experience and thus not report a stronger rubber-hand illusion as they do not meet the necessary cognitive requirements.

More susceptible individuals, by contrast, are expected to also develop impressions of some tactile sensations that originate from the fake hand. Such experiential effects are relatively demanding as they require uniting contradicting visual and tactile information into a single percept. Even more demanding are impressions that require a recalibration of the mental representation of one's body image, such as experiencing the fake hand as part of one's body.

Only those few individuals that are extremely susceptible to the illusion are expected to encounter visual changes in the appearance of the fake hand, as this is presumed to require a prolonged feeling of ownership over the fake hand (see Lewis \& Lloyd, 2010). These people will not only report altered visual perceptions, but are also expected to experience a sense of ownership over the fake hand, a relocation of felt touch, and impressions of strangeness and confusion.

Thus, if our assumption of an invariant and transitively ordered set of experiential effects holds, then we can predict how a person experiences, and thus reports on, the rubber-hand illusion on the basis of an estimate of his or her susceptibility to the illusion. The specific transitive order of increasingly demanding experiential effects ultimately defines the rubber-hand illusion. In our conception, all experiential effects related to the rubber-hand illusion fall onto a single continuum rather than being organized into a set of multiple dimensions (cf. Longo et al., 2008). Since multidimensional conceptions of the rubber-hand illusion require a matching number of individual abilities, our model offers a more parsimonious account of the individual differences in people's reports about the illusion.

\subsection{A Rasch model implementation}

The hypothesized relationship between a person's self-reported experiences, his or her susceptibility to the illusion, and the presumed cognitive demand behind each specific experiential effect can be 
described mathematically with the Rasch model (for details, see e.g., Bond \& Fox, 2007):

$\ln \left(\frac{\mathrm{p}\left(x_{n i}=1\right)}{1-\mathrm{p}\left(x_{n i}=1\right)}\right)=\theta_{n}-\delta_{i}$

The Rasch model describes the natural logarithm of the odds of reporting a certain experiential effect $i\left[\mathrm{P}\left(x_{n i}=1\right)\right]$ as an additive function of a person $n$ 's susceptibility $\left(\theta_{n}\right)$ and the cognitive demand $\left(\delta_{i}\right)$ assumed behind that particular experience. Both parameters in this equation (susceptibility and cognitive demand) are estimated by means of maximum-likelihood estimation. Susceptibilities of persons and the cognitive demands behind the various self-report items are expressed in log odd units (also called logits). For a specific experiential effect $i$ to have a 50\% chance to be developed, and thus to be reported, its cognitive demand (e.g., $\delta_{i}=1$ ) has to be matched numerically with an equivalent amount of a person $n$ 's susceptibility $\left(\theta_{n}=1\right)$.

The Rasch model, however, is not only descriptive, but also prescriptive. It requires self-report items to be ordered invariantly and transitively across persons, and persons to be ordered invariantly and transitively across items. Thus, if a person reports, for example, four out of 10 experiential effects, the Rasch model also prescribes which four should be experienced: They should be the four least demanding ones. And for the most demanding experiential effects, we expect to exclusively find such reports from the most susceptible persons.

These formal Rasch model expectations can be tested empirically against the observed data. Provided the responses are ordered from highly to lowly susceptible persons, the model anticipates the following response string for an averagely demanding experiential effect: 111101010000. In this response string, a one indicates that a person agreed to have encountered the experience, and a zero that a person did not. The first four individuals all have high susceptibilities, so all four are likely to develop, and thus report, this experience. The next four individuals have a susceptibility that approaches numerically the cognitive demand of this experiential effect. As a result, some of them will agree, but others may not. Finally the last four individuals have such a low susceptibility that all are very unlikely to develop this experience (hence all zeros should be observed).

The mean square (MS) statistic is commonly used to test the match between model-predicted and observed response patterns. The MSvalue is the weighted average of the squared standardized residuals, in which each residual is weighted by its variance (e.g., Bond \& Fox, 2007). The model-predicted response pattern (e.g., 111101010000,) yields a $M S$-value of $M S=1.00$. Excessively high $M S$-values can be expected when the observed response string opposes the Rasch model prediction, for example when the likelihood of reporting an experiential effect increases with diminishing susceptibility (e.g., 000000111111). In contrast, the MS-value would be smaller than 1.00 for an item with a deterministic response pattern (i.e., 111111000000). In such a case, the model prediction-to-data fit is better than what one would anticipate with a probabilistic model. MS-values below 1.00 do not really challenge the Rasch model prediction, but can be used to improve one's measurement instrument.

The invariance assumption should be sufficiently met in order to map both persons and experiential effects on a single scale (see Fig. 1), and thus to compare individuals meaningfully in respect to their susceptibility to the rubber-hand illusion. For assessing item fit, $M S$-values up to 1.20 are considered good, and MS-values below 1.50 are considered acceptable (Wright \& Linacre, 1994). Standardized fit statistics can be used with persons. By chance alone, $2.5 \%$ of the individuals will have a $t$-value of $t \geq 1.96$. As a rule of thumb, $5 \%$ of poorly fitting persons are considered acceptable for most purposes.

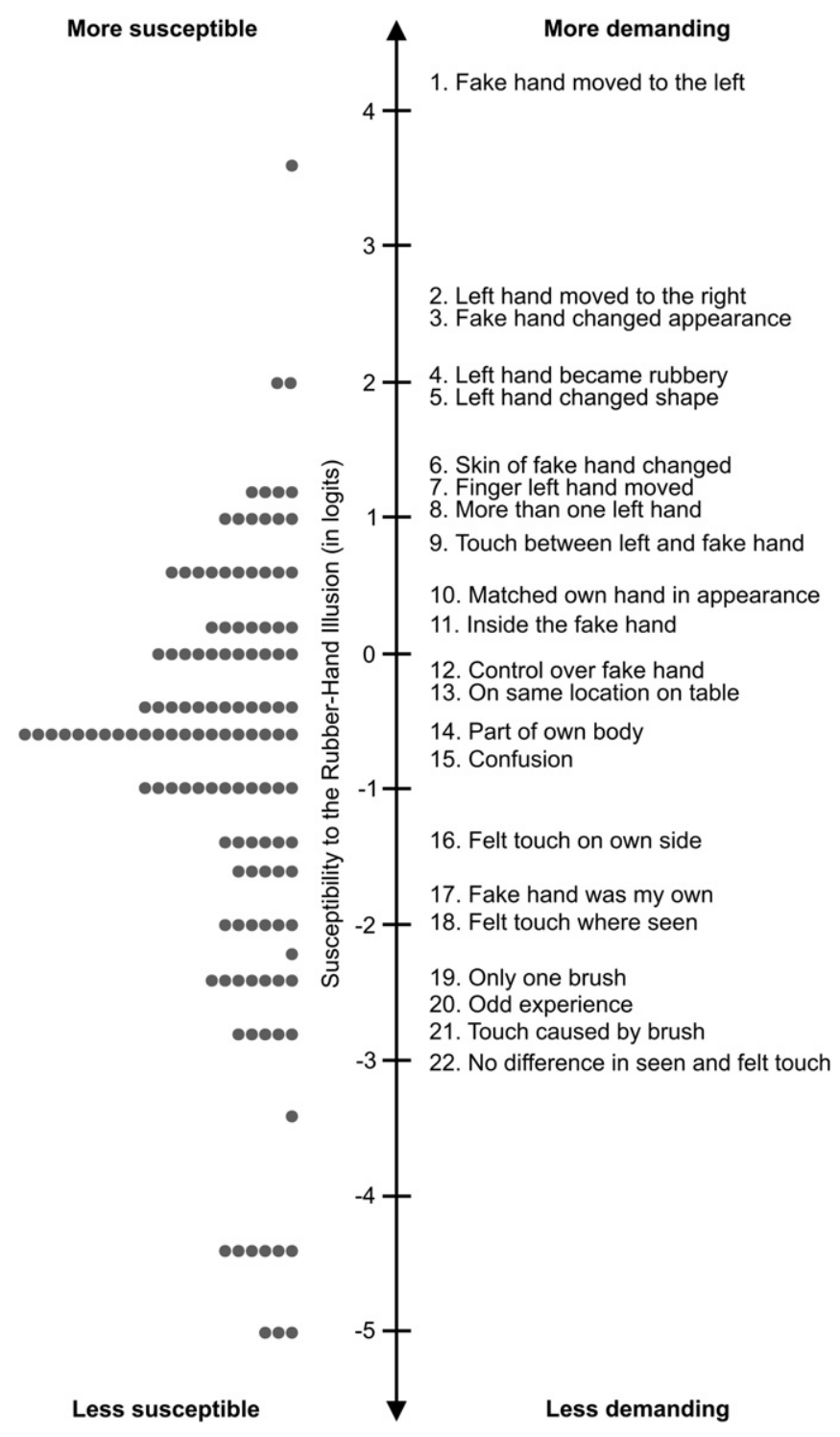

Fig. 1. Item-person map for Experiment 1. The estimates of each participant's susceptibility and of the cognitive demand linked with each experiential effect mapped onto a single scale. Each dot represents a single individual.

\section{Experiment 1}

In this first experiment, we model reports of 22 experiential effects presumed to be related to the rubber-hand illusion, based on the Botvinick and Cohen (1998) instrument and other sources, as a function of individual susceptibility and of the cognitive demand behind each experience. A successful Rasch model test will, on the one hand, corroborate the hypothesis that individuals can be reliably differentiated with regard to their susceptibility to the rubber-hand illusion. On the other hand, it confirms a transitive and invariant order of the 22 experiential effects for all persons involved in the experiment. Additionally, we investigate the relationship between individual susceptibility and proprioceptive drift. We predict that people who incorporate the fake hand more strongly into their body image will also show a more pronounced proprioceptive drift (cf. Tsakiris \& Haggard, 2005).

\subsection{Methods}

\subsubsection{Participants}

Our sample of participants was drawn from students and employees of the Eindhoven University of Technology, Eindhoven, The 
Netherlands. One hundred and twenty-seven persons participated in this experiment, 100 (79\%) of whom were male. Their mean age was 24.7 ( $S D=6.5$; range 19 to 65 ); $73 \%$ of our participants were right handed and 14\% were ambidextrous (according to the Dutch handedness scale; van Strien, 1992). All participants received coffee and cake as compensation.

\subsubsection{Procedure and apparatus}

Participants were seated in a chair with their left hand on a table in front. First, the experimenter obtained, for each participant, the pre-exposure difference between actual and felt location of the left hand. For this, the experimenter asked the participant to close his or her eyes. Then, a small platform ( 25 by 80 by $9 \mathrm{~cm}$ ) was placed over the participant's left hand (see Fig. 2A). A string was mounted over the length of the platform to which a small block was attached. Next, the experimenter guided the participant's right hand to this block. After that, the experimenter instructed the participant to slide the block over the platform (starting from the participant's outermost right-hand side) to indicate the felt position of his/her middle finger of the left hand. The difference between actual and felt position was consecutively assessed three times. It was coded with a positive sign when the felt position was biased towards the participant's right-hand side, and with a negative sign when the felt position was biased beyond the left hand's actual position.

After the platform was removed, participants were asked to move their hands as if conducting an orchestra, while the experimenter placed a fake hand in front of them. As a fake hand, a cosmetic prosthesis of a man's left hand was used that was realistic in terms of skin texture, color, and shape. With each participant, the rubber-hand illusion was then induced in a single five minute trial. For this, participants were asked to place their left hand back on the table (at a lateral distance of $30 \mathrm{~cm}$ from the fake hand) and to keep it motionless (see Fig. 2B). Next, the experimenter concealed the participant's left hand from his or her view by placing a screen between the left and the fake hand. Subsequently, the experimenter stroked and tapped the middle and index fingers of both hands, simultaneously and in synchrony, by means of two brushes.

After the induction of the illusion, participants were instructed to continue to keep their left hand motionless on the table. Subsequently, the experimenter obtained, for each participant, the post-exposure difference between actual and felt location of the left hand, using the same procedure as before. Finally, the participants were asked to fill out a questionnaire.

\subsubsection{Measures}

2.1.3.1. Self-reports on personal impressions. The self-report items consisted of 22 statements presumed to describe experiential effects related to the rubber-hand illusion, such as "It felt as if the fake hand was part of my own body". All items were presented to participants in Dutch. Thirteen of these items were adopted from previously used instruments (e.g., Armel \& Ramachandran, 2003; Botvinick \& Cohen, 1998; Haans et al., 2008; IJsselsteijn, de Kort, \& Haans, 2006; Longo et al., 2008; Mussap \& Salton, 2006). For a complete list of the 22 statements, see Table 1 . Participants were asked to indicate whether they agreed or disagreed with these statements by means of five response categories, labeled "disagree", "slightly disagree", "neutral”, "slightly agree", and "agree”. For all participants and across all 22 items, there were only two missing responses $(<0.1 \%)$.

To keep the subsequent calibrations straightforward in their interpretation, we recoded the individual responses to the 22 impressions into a dichotomous format. For this purpose "disagree", "slightly disagree", and "neutral" were collapsed into "refute", and "slightly agree" and "agree" into "assert". Such dichotomization is not expected to affect the meaning of the personal statements (Linacre, 2009). The Rasch model test was performed using the Facets software (Linacre, 2010). The Facets software employs a joint maximum likelihood procedure to estimate (a) each participant's susceptibility, and (b) the presumed cognitive demand behind each of the 22 experiential effects.

2.1.3.2. Proprioceptive drift. Proprioceptive drift is the difference of two differences: the pre-exposure difference subtracted from the post-exposure difference between actual and felt position of a participant's left hand (Tsakiris \& Haggard, 2005). Since pre- and post-exposure differences were each assessed three times, three proprioceptive drift estimates could be calculated for each participant. For this purpose, we subtracted the first pre-exposure difference from the first post-exposure difference, the second pre-exposure difference from the second post-exposure difference etc. The average of these three estimates was used in our analyses. The reliability (Cronbach's alpha) of the three estimates of proprioceptive drift was $\alpha=.89$.

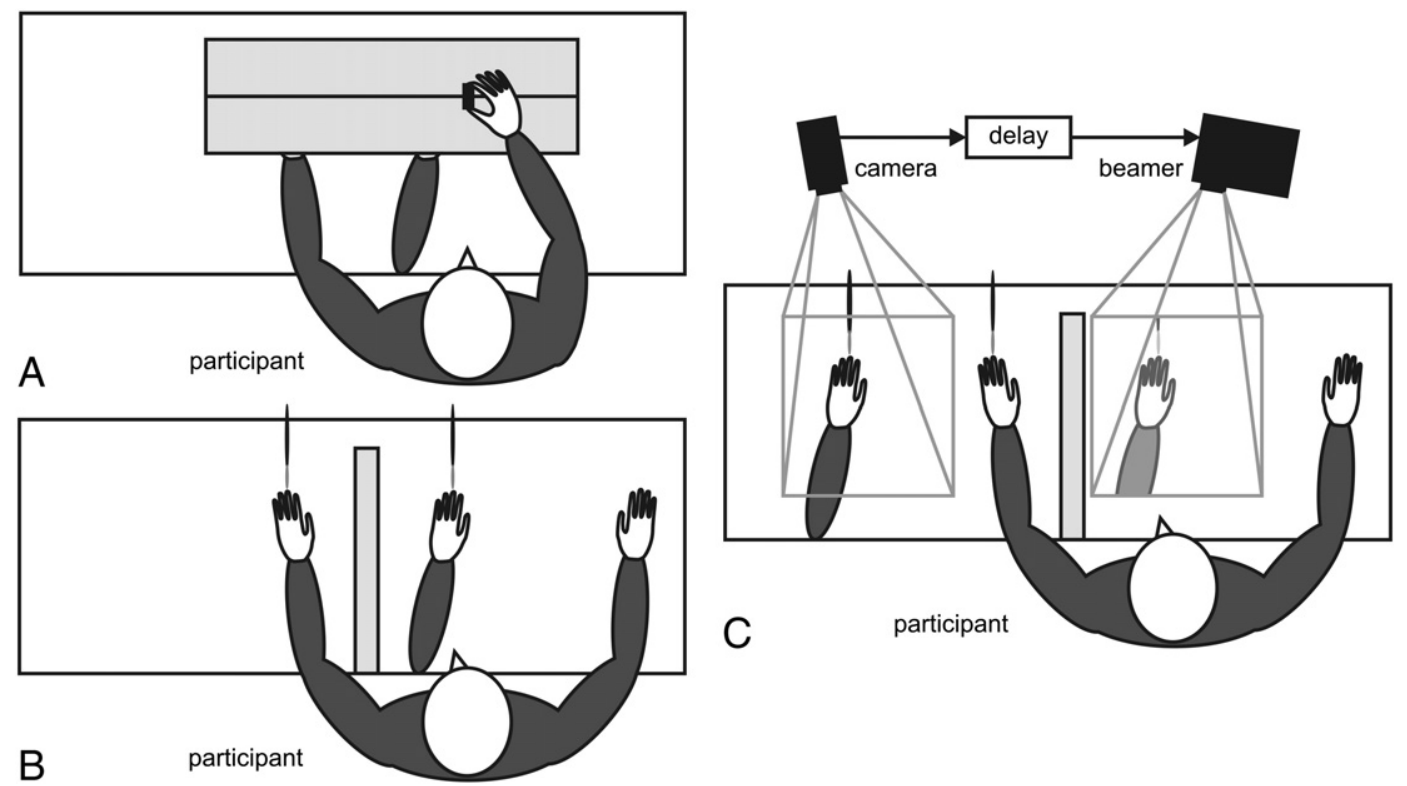

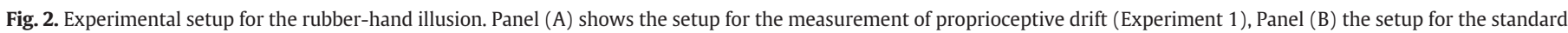
rubber-hand illusion (Experiment 1), and Panel (C) the setup for the mediated rubber-hand illusion (Experiment 2). 
Table 1

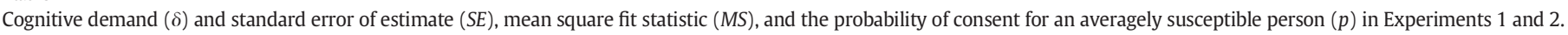

\begin{tabular}{|c|c|c|c|c|c|c|}
\hline \multirow[t]{2}{*}{ Experiential effect } & \multicolumn{3}{|l|}{ Experiment 1} & \multicolumn{3}{|l|}{ Experiment 2} \\
\hline & $\delta(S E)$ & MS & $p$ & $\delta(S E)$ & MS & $p$ \\
\hline 1 Sometimes, it appeared as if the fake hand moved to my left hand side. & $4.16(.73)$ & 1.11 & .04 & $3.77(.71)$ & 1.17 & .04 \\
\hline 2 Sometimes, it felt as if my left hand moved towards my right hand side. & $2.57(.39)$ & 1.18 & .16 & $1.89(.46)$ & 0.94 & .13 \\
\hline 3 Sometimes, the shape of the fake hand appeared to change. & $2.57(.39)$ & 0.91 & .16 & $0.95(.38)$ & 0.96 & .21 \\
\hline 4 Sometimes, it felt as if my left hand turned rubbery. & $1.96(.32)$ & 1.12 & .25 & $0.75(.37)$ & 1.15 & .24 \\
\hline 5 Sometimes, it felt as if the shape of my left hand started to change. & $1.95(.32)$ & 1.00 & .26 & $2.54(.52)$ & 1.18 & .08 \\
\hline 6 Sometimes, the skin properties of the fake hand (i.e., color or texture) appeared to change. & $1.24(.26)$ & 0.94 & .41 & $0.07(.34)$ & 0.78 & .33 \\
\hline 7 When a finger of the fake hand moved, I sometimes felt the finger of my left hand moving as well. & $1.17(.25)$ & 1.08 & .43 & $0.31(.35)$ & 1.08 & .30 \\
\hline 8 Sometimes, it felt as if I had more than one left hand. & $1.11(.25)$ & 1.12 & .44 & $2.36(.50)$ & 1.27 & .09 \\
\hline 9 Sometimes, it appeared as if the touch I felt originated from somewhere between my own hand and the fake hand. & $0.71(.23)$ & 0.95 & .54 & $1.76(.44)$ & 1.19 & .14 \\
\hline 10 Sometimes, the fake hand began to match my own hand in appearance. & $0.31(.22)$ & 0.86 & .64 & $-0.44(.33)$ & 1.11 & .41 \\
\hline 11 Sometimes, it felt as if my left hand was inside the fake hand. & $0.12(.21)$ & 0.90 & .68 & $1.89(.46)$ & 0.95 & .13 \\
\hline 12 Sometimes, it felt as if I had complete control over the fake hand: I could have moved the fake hand if I wanted to. & $-0.14(.21)$ & 0.97 & .73 & $0.07(.34)$ & 0.74 & .33 \\
\hline 13 Sometimes, it felt as if my left hand and the fake hand were on the same location on the table. & $-0.27(.21)$ & 0.91 & .76 & $0.75(.37)$ & 0.91 & .24 \\
\hline 14 Sometimes, it felt as if the fake hand was part of my own body. & $-0.66(.21)$ & 0.87 & .82 & $-0.09(.34)$ & 0.70 & .35 \\
\hline 15 Sometimes, I became confused about what I saw and felt. & $-0.87(.21)$ & 0.99 & .85 & $-2.01(.30)$ & 1.45 & .67 \\
\hline 16 Sometimes, it appeared as if I felt the touch on my side of the wooden screen (i.e., on the side of the fake hand). & $-1.37(.22)$ & 0.95 & .90 & $0.07(.34)$ & 1.13 & .33 \\
\hline 17 Sometimes, it felt as if the fake hand was my own hand. & $-1.78(.23)$ & 0.78 & .93 & $-0.99(.31)$ & 0.68 & .50 \\
\hline 18 Sometimes, it appeared as if I felt the touch on the location where I saw the fake hand being touched. & $-1.97(.23)$ & 1.09 & .95 & $-1.83(.30)$ & 1.03 & .64 \\
\hline 19 I continuously felt as if the touches on my fingers and the touches on the fake hand were caused by two different brushes. & $-2.38(.25)$ & 1.06 & .96 & $-3.22(.30)$ & 0.93 & .82 \\
\hline 20 I did not experience anything odd. & $-2.51(.26)$ & 1.04 & .97 & $-3.34(.30)$ & 1.31 & .83 \\
\hline 21 Sometimes, it felt as if the touches on my fingers were caused by the brush touching the fake hand. & $-2.88(.28)$ & 1.06 & .98 & $-2.76(.30)$ & 0.73 & .77 \\
\hline 22 I continuously noticed clear differences between the touches I saw on the fake hand, and the touches I felt. & $-3.04(.29)$ & 1.06 & .98 & $-2.53(.30)$ & 0.99 & .74 \\
\hline
\end{tabular}

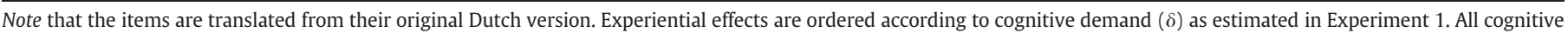

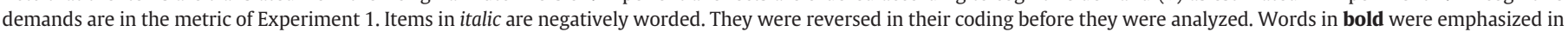

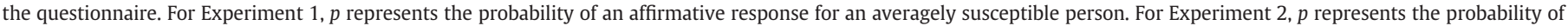
affirmation for an averagely susceptible person in the synchronous (i.e., $51 \mathrm{~ms}$ delay) stroking condition.

\subsection{Results and discussion}

In the following sections, we will first present the results of the model test, including the person and item fit statistics. Second, we compare each participant's susceptibility estimate with his or her proprioceptive drift.

\subsubsection{Model test}

The estimated cognitive demands behind each of the 22 experiential effects are reported in Table 1 . All items fit the model sufficiently with $M S$-values $\leq 1.20$. The individual susceptibilities to the rubber-hand illusion were estimated with a reliability of .82 . The average susceptibility was $M=-0.88$ logits $(S D=1.59$; range -5.64 to 3.54 ; see Fig. 1$)$. For only four $(3.1 \%)$ of the 127 participants, the model prediction did not fit the data as indicated by a $t$-value of $t \geq 1.96$. Empirically, the Rasch model explained $48.1 \%$ of the variance in the data (for computational details, see Linacre, 2003). Because the Rasch model estimates probabilities for discrete events (i.e., whether a person claims to have encountered a certain experiential effect or not), substantial quantization variance is to be expected. If the model would fit perfectly, then $52.9 \%$ of the overall variance would be quantization variance. The empirical proportion of unexplained variance (i.e., 51.9\%) was thus highly similar to the proportion of quantization variance one would expect with perfect data-to-model fit. In addition, we performed a principal component analyses on the standardized residuals (i.e., the data not explained by our model) to explore the possibility for another unaccounted factor in the data (Linacre, 1998). As an additional factor would only result in a trivial increase of $4.6 \%$ in the proportion of explained variance, we conclude that our set of 22 items taps into a single factor only. Taken together these statistics demonstrate that the observed data fit the model-prediction rather well, thereby confirming our hypothesis that the presumed cognitive demands behind each of the 22 experiential effects are independent of the individual taking the test.

For a more stringent test of this hypothesis, we split our sample in half and estimated the cognitive demand related with each experiential effect twice: once for persons with even and once for persons with odd identification numbers. Consistent with our hypothesis of person-independent cognitive demands, the two estimates of the 22 cognitive demands were highly similar with $r=.94$, and $p<.01$ (see Fig. 3A). Subsequently, we used the cognitive demands as estimated with the even sample, to test the data-to-model fit in the uneven sample. At least some misfit is to be expected as the cognitive demands are now estimated based on the responses of only 63 participants. Nevertheless, the responses to all but two of the items fitted the model reasonably well with $M S \leq 1.30$ : "it appeared as if the fake hand moved to my left hand side" (Item $1 ; M S=5.48$ ) and "It felt as if my left hand turned rubbery" (Item $4 ; M S=3.48$ ). At the same time, for only three $(4.7 \%)$ out of 64 participants did the responses not fit the Rasch model. In other words, there is sufficient model prediction-to-data fit, even when the cognitive demands are estimated from an independent sample.

\subsubsection{Susceptibility and proprioceptive drift}

Our participants, on average, demonstrated a proprioceptive drift of $M=1.9 \mathrm{~cm}$ ( $S E=0.4$; range -5.7 to 17.6$)$. We found a small, but significant, correlation between individual susceptibility and proprioceptive drift $(r=.20, p=.03)$. Even after correction for measurement error attenuation (cf. Charles, 2005), the correlation remained small, with $r_{\text {corr }}=.23$, indicating an overlap in variance of about $5.3 \%$. As expected, proprioceptive drift increased with individual susceptibility, thereby providing support for the construct validity of our susceptibility measure.

\section{Experiment 2}

When taking a mathematical exam, distracting background noises or poor illumination of the room will obstruct even the most proficient students in realizing their full potential. Similarly, one can obstruct the extent to which people can incorporate a fake hand into their body image by manipulating features of the experimental setup in which the rubber-hand illusion is induced (e.g., the amount of asynchrony in the visuotactile stimulation). In other words, to predict how people experience the rubber-hand illusion, we need to consider three factors rather than two: Individual susceptibility, the cognitive demand behind the various experiential effects, and the constraints imposed by the experimental setup in which the rubberhand illusion is elicited. 
A

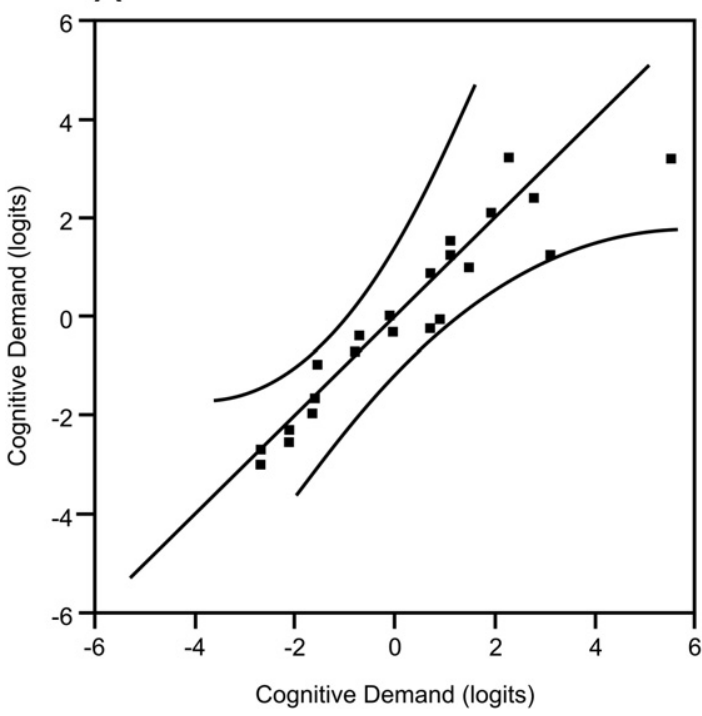

$\mathrm{B}$

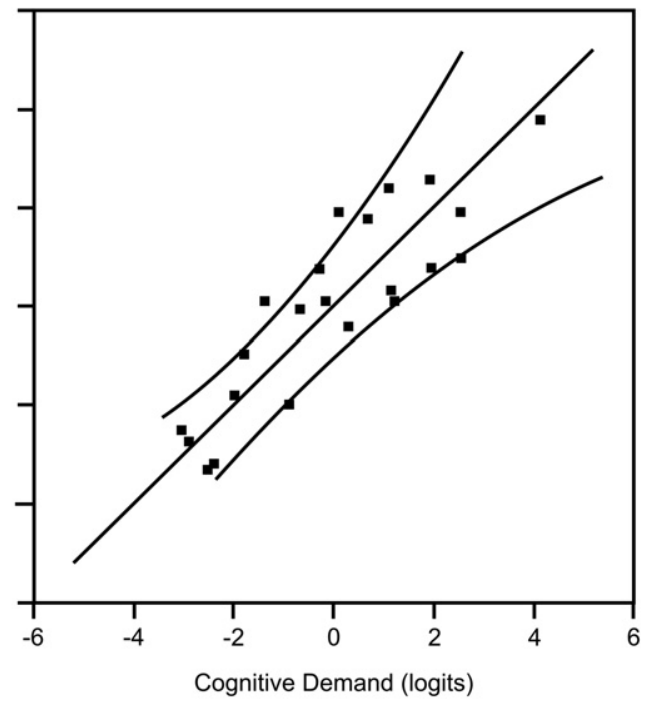

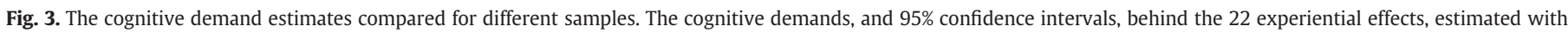

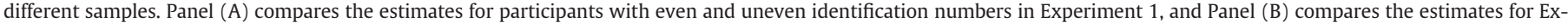
periments 1 and 2 .

For this second experiment, we manipulate two features of the experimental setup: the extent of asynchrony and the type of stimulation (tapping vs. stroking). Research suggests that incorporation of a rubber hand into the body image is largely dependent on sensorimotor integration, and thus on the capability of the central nervous system to extract correlations between the various sensory modalities (e.g., Armel \& Ramachandran, 2003). By introducing a delay between the stimulation of the fake hand and that of the participant's concealed hand, it becomes more difficult for the central nervous system to extract a strong correlation between seen and felt stimulation. As a result, this asynchrony obstructs the incorporation of the fake hand in the body image (e.g., Ehrsson et al., 2004; Shimada, Fukuda, \& Hiraki, 2009; Tsakiris \& Haggard, 2005). By contrast, we expect that the rubber-hand illusion can be facilitated by stroking rather than tapping the fingers of the fake hand and the participant's concealed hand. If the fingers of the real and fake hand are stroked rather than tapped, then there is more information in the seen and felt stimulation that can covary: not only information regarding location and duration, but also regarding speed and direction. Such information-rich stimulation allows the central nervous system to extract a stronger correlation between the two modalities, which, in turn, is expected to result in a more vivid rubber-hand illusion.

Eq. (2) gives a many-facet Rasch-type model that includes estimates for the contextual constraints imposed by the experimental setup (for more details see Linacre, 2002; also Bond \& Fox, 2007):

$\ln \left(\frac{p\left(x_{\text {nidt }}=1\right)}{1-p\left(x_{\text {nidt }}=1\right)}\right)=\theta_{n}-\left(\delta_{i}+\lambda_{d}+\lambda_{t}\right)$.

In this model, the natural logarithm of the odds that person $n$ reports a certain experiential effect $i$ (e.g., the fake hand feels as $n$ 's own) is governed by four factors: $n$ 's susceptibility to the illusion $\left(\theta_{n}\right)$, the specific cognitive demand required to develop that experience $i\left(\delta_{i}\right)$, and the constraints imposed by the amount of delay $d$ $\left(\lambda_{d}\right)$ and the type of stimulation $\left(\lambda_{t}\right)$ in the visuotactile stimulation.

\subsection{Method}

\subsubsection{Participants}

Our sample of participants was drawn from students and employees of the Eindhoven University of Technology, Eindhoven,
The Netherlands. Twenty-four persons participated in this experiment, 18 (75\%) of which were male and all were sensitive to the rubber-hand illusion (which was assessed a few days prior to the actual experiment). Their mean age was 21.8 ( $S D=2.4$; range 18 to 28 ); $83 \%$ of the participants were right handed and $13 \%$ were ambidextrous (as based on the Dutch Handedness scale; van Strien, 1992). All participants received $€ 7.00$ as compensation.

\subsubsection{Experimental design and apparatus}

A six (delay between felt and seen stimulation in steps of $100 \mathrm{~ms}$ ) by two (tapping vs. stroking) within-subject design was implemented. To reduce the number of trials per participant, the six delay conditions were employed with each participant only once. As a result, each person participated in only 6 of the 12 experimental conditions. The order of the delays was randomly assigned to the participants. At the same time, we controlled that each delay condition was presented equally often in the first trial of each session. Whether a participant would receive strokes or taps during a certain delay condition was determined by means of a rotated-judgment plan (for more details, see Linacre, 2002).

\subsubsection{Procedure and apparatus}

The rubber-hand illusion was elicited in six trials. To enable a reliable delay between seen and felt stimulation, a technologically mediated implementation of the rubber-hand illusion was used (for technical details, see IJsselsteijn et al., 2006). In this procedure, participants were not looking at the fake hand directly, but at a video projection of the fake hand (see Fig. $2 \mathrm{C}$ ). The projection of the fake hand and its stimulation was delayed by means of an Evertz7700 delay unit placed between the camera and the beamer. The delay intrinsic in the camera and beamer setup was $51 \mathrm{~ms}$ (i.e., phase shift estimated with a pulsating LED and two Phidgets Precision Light Sensor modules sampled at $10 \mathrm{kHz}$, connected to a Meilhaus Redlab series 1608 data acquisition module). The six asynchrony conditions thus consisted of $51,151,251,351,451$, and $551 \mathrm{~ms}$ delay respectively. The experimenter was unaware of the degree of asynchrony in each trial. To ensure a constant interval between subsequent strokes or taps, the experimenter used a headset through which a tone was played at a one second interval. After each trial, participants completed a questionnaire. 


\subsubsection{Measures}

The extent to which participants incorporated the fake hand into the body image was assessed with the same 22 self-report items as in Experiment 1. We processed these items in the same manner as described in Experiment 1. There were no missing responses.

\subsection{Results and discussion}

We will report the results of the model test in two parts. First, we detail the estimation of the individual differences in susceptibility and of the presumed cognitive demand behind the 22 experiential effects. Second, we present the estimation of the two contextual constraints: asynchrony and type of stimulation.

\subsubsection{Susceptibility and cognitive demand}

The cognitive demand estimates for each of the 22 experiential effects are reported in Table 1 . This time, all but three items fitted the model with $M S$-values below 1.20 . The three items with $M S>1.20$ were: Item 8 "Sometimes, it felt as if I had more than one left hand" $(M S=1.27)$, Item 15 "Sometimes, I became confused about what I saw and felt" ( $M S=1.45)$, and Item 20 "I did not experience anything odd" ( $M S=1.31$; see Table 1$)$. In addition, we correlated the two estimates of the 22 cognitive demands from Experiments 1 and 2 (see Fig. 3B). Again, we found a high correlation between the two estimates, with $r=.88$ and $p<.01$.

The individual differences in susceptibility to the rubber-hand illusion were estimated with a reliability of .95 . The average susceptibility was $M=-1.66$ logits ( $S D=1.28$; range -4.10 to 1.05 ). This time, for $2(8.3 \%)$ out of 24 participants the model prediction did not fit the data, indicated by significant $t$-values of $t>1.96$. These statistics corroborate our findings in Experiment 1: The data fit the model-prediction quite acceptably, thereby confirming our hypothesis that each experiential effect related to the rubber-hand illusion involves a specific and person-independent cognitive demand.

\subsubsection{Contextual constraints}

The estimates for the constraints caused by each of the six delay conditions are depicted in Fig. 4. The different delay conditions had a significant effect on the development of the rubber-hand illusion: $\chi^{2}(5, N=$ 24 ) $=169.4, p<.01$ (for computational details, see Schumacker \& Lunz, 1997). As expected, we found that it became more and more difficult to incorporate the fake hand into the body image when asynchrony between seen and felt stimulation increased. For example, an average person had about $50 \%$ chance of developing, and thus reporting, a sense of ownership toward the fake hand (Item 17 in Table 1), when there was only $51 \mathrm{~ms}$ delay between seen and felt simulation (in the stroking condition; see Fig. 4). By contrast, a $451 \mathrm{~ms}$ delay reduced this probability to $15 \%$. Similarly, the average person had about $33 \%$ chance of reporting a sense of agency (Item 12 in Table 1) with 51 ms delay, but this probability was only $8 \%$ with delays of $451 \mathrm{~ms}$ or more (in the stroking condition). Asynchrony can thus be seen as a contextual constraint that increases the cognitive demand behind experiencing the illusion. Based on fit statistics, we can also conclude that the extent of this obstruction can be accurately estimated (all six estimates resulted in MS-values below 1.20).

The extent to which type of stimulation constrained the development of the illusion was estimated at 0.20 logits $(S E=0.07)$ for tapping, and -0.20 logits $(S E=0.07)$ for stroking. As expected, tapping (i.e., information-poor stimulation), compared to stroking, significantly constrained the development of the rubber-hand illusion: $\chi^{2}(1, N=24)=15.0$, and $p<.01$. This implies, for example, that an average person had a $10 \%$ lower chance of developing, and thus reporting, a sense of ownership over the fake hand (Item 17 in Table 1) in the tapping as compared to the stroking condition (with 51 ms delay). Again, the fit statistics for the tapping and the stroking effect estimates were good, with MS-values below 1.20.

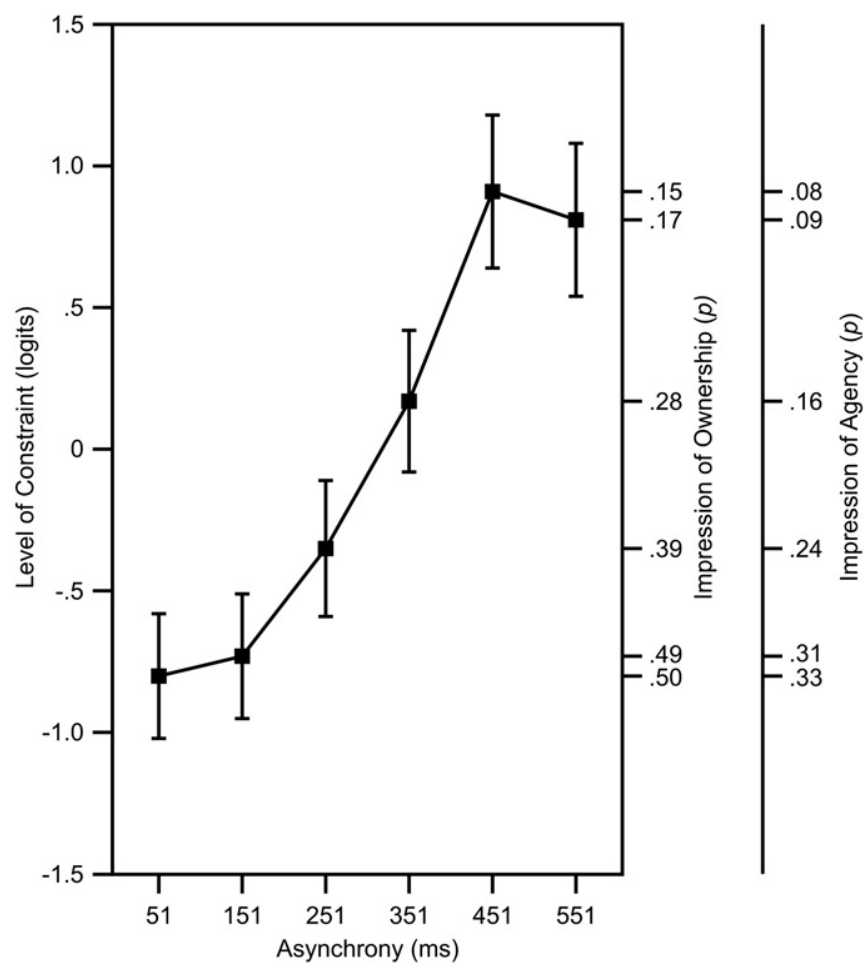

Fig. 4. Estimated contextual constraint as a function of asynchrony. Error bars depict 95\%-confidence intervals. The rightmost y-axes give the probability of an averagely susceptible person in the stroking condition claiming that "the fake hand felt as my own" (ownership; Item 17 in Table 1) and that "it felt as if I had complete control over the fake hand" (agency; Item 12 in Table 1) as a function of delay.

The interaction between asynchrony and type of stimulation was not found to be significant: $\chi^{2}(12, N=24)=6.1$, and $p=.91$.

\section{General discussion}

In two experiments, we were successful in predicting people's responses to 22 items about experiential effects presumed to be related to the rubber-hand illusion (e.g., a sense of agency over the fake hand). For that, we used estimates of people's susceptibility to the illusion, estimates of the presumed cognitive demand behind each experiential effect, and estimates of the constraints intentionally imposed through manipulations in the experimental setup (i.e., latency between seen and felt touch, and type of touch).

The 22 experiential effects included in the present paper are but a preliminary set based mostly on existing measures and qualitative research. Nevertheless, 17 out of the 22 self-report items fitted the model rather well with MS-values below 1.20 in all analyses. Responses to five statements seem problematic at least in some of the analyses: "The fake hand moved to my left hand side" (Item 1; see Table 1), "My left hand turned rubbery" (Item 4), "I had more than one left hand" (Item 8), "I became confused about what I saw and felt" (Item 15), and "I did not experience anything odd" (Item 20). These statements are either comparatively poor indicators of the rubber-hand illusion or problematic in their phrasing. With respect to the latter two statements, it might also be that the asynchrony between seen and felt stimulation in Experiment 2 in and of itself caused impressions of confusion.

Overall, our model tests confirm our hypothesis that the various experiential effects related to the rubber-hand illusion (e.g., the fake hand felt as one's own) can be transitively and invariantly ordered with respect to how likely they are reported. In our conception, this ordering reflects that the cognitive demand behind each experiential effect is highly similar for all people included in the experiments. As a result, the large individual differences in people's reports about the 
illusion can be explained by a single ability. This susceptibility to the rubber-hand illusion reflects the extent to which a person can activate certain cognitive and sensorimotor processes, or inhibit others, which allows the incorporation of a fake hand into the body image. People with comparable levels of susceptibility develop and, thus report, similar experiences.

The existence of large differences in the ease with which various experiential effects can be developed poses a problem for conventional factor analysis (e.g., Longo et al., 2008; also Longo, Schüür, Kammers, Tsakiris, \& Haggard, 2009). Large differences in item difficulty may give rise to spurious dimensions (so-called difficulty factors; e.g., Gorsuch, 1997). Indeed, our analysis indicates that the 22 self-report items tap into a single dimension only. Our instrument, however, did not completely overlap with the one used by Longo et al. $(2008,2009)$ which also contained statements regarding the loss of one's own concealed hand (e.g., it seemed like my own hand had disappeared). Recent research suggests that incorporation of a fake hand into the body image and changes in the perception of one's own hand might indeed be independent consequences of the rubber-hand illusion (cf. Kammers et al., 2009).

Our results confirm a single underlying dimension in the Botvinick and Cohen (1998) instrument. Since Rasch model-based estimates correlate highly with aggregated scores (i.e., by summing or averaging persons' responses), our results lend credence to the use of such aggregates to measure the strength of the rubber-hand illusion (e.g., Haans et al., 2008).

With our measure, we were able to reliably differentiate people with respect to their susceptibility to the illusion $(\mathrm{rel} \geq .82)$. More research is needed to theoretically explain the large differences in people's susceptibility to the illusion. Correlating susceptibility with other aspects of the individual's psychological make-up, such as body image instability (cf. Burrack \& Brugger, 2005), may provide further evidence for the validity of our model. We did find a small but significant correlation between individual susceptibility, and thus the strength of the illusion, and proprioceptive drift (with $r_{\text {corr }}=.23$ ). The fact that this correlation is small, confirms previous findings that proprioceptive drift has limited validity as a measure of the rubber-hand illusion (cf. Rohde et al., 2011). At the same time, the significant correlation supports the view that the recalibration of proprioception might facilitate the development of the illusion (cf. Holmes et al., 2006).

The proposed model also has proven useful in describing the effects of situational constraints imposed by the experimental setup on the extent to which people incorporated the fake hand into the body image. As anticipated, we found that an asynchrony between seen and felt stimulation hindered the development of the rubber-hand illusion (cf. Botvinick \& Cohen, 1998; Tsakiris \& Haggard, 2005). Specifically, longer delays result in less convincing rubber-hand illusions, and thus by inference weaker body image incorporations (see Fig. 4; for a similar finding, see Shimada et al., 2009). Additionally, stroking the fingers of the fake hand, compared to tapping, was found to facilitate the development of the illusion. We expected such an effect, because stroking is richer in information than tapping. Alternative explanations for the tapping-stroking difference in eliciting the rubber-hand illusion must, however, be considered. A stroke might stimulate other cutaneous receptors than a tap. Further research is needed to understand what mechanisms explain best the observed tapping-stroking difference. The least we can say is that our findings provide empirical support for the validity of our model.

There were two limitations to the present experiments. First, we did not randomize the order of the self-report items in our questionnaires. This might have affected our participants' responses in a structured manner, thereby strengthening the presumed invariance in the order of the experiences. However, recent evidence suggests that the invariance hypothesis is still supported when the 22 items are presented in a randomized order (Haans \& IJsselsteijn, in preparation). A second limitation of the present study is that there is still little theoretical ground on which to base the item selection process. Ideally, item selection should be based on theoretical models about the cognitive and neural underpinnings of the illusion. To our knowledge, however, only one such model has been formulated so far (see Makin, Holmes, \& Ehrsson, 2008).

Despite these limitations, our model seems nevertheless promising. It explains individual differences in reports about experiences related with the rubber-hand illusion in a parsimonious way. It provides a solid rationale for considering the individual variations in subjective reports associated with the rubber hand illusion as data rather than noise, reflecting a single underlying dimension of increasingly demanding experiences. Thus, our model can be used as a starting point to explore the origins of individual differences in susceptibility, or to explore the specific cognitive requirements behind the various experiential effects related to the rubber-hand illusion. As such, our research facilitates the development of a theoretical model of the rubber-hand illusion.

\section{Acknowledgments}

This research was supported by the JF Schouten School for User-System Interaction Research at Eindhoven University of Technology, Eindhoven, The Netherlands. We gratefully acknowledge Otto Bock Benelux B.V. for donating the materials used in the present study. We thank Pieter Custers, Berrie Duijx, Thomas van Erp, Frits Koster, Ludo van Leeuwen, René Segers, and Carmen Wijermans for their assistance in conducting the experiments, and Martin Boschman for assisting us with the laboratory setup. We also thank Nicholas Holmes and Daniel Lakens for their comments on earlier drafts of this manuscript.

\section{References}

Armel, K. C., \& Ramachandran, V. S. (2003). Projecting sensations to external objects: Evidence from skin conductance response. Proceedings of the Royal Society of London B, 270, 1499-1506.

Bond, T. G., \& Fox, C. M. (2007). Applying the Rasch model: Fundamental measurement in the human sciences (2nd ed.). Mahwah, NJ: Erlbaum.

Botvinick, M. (2004). Probing the neural basis of body ownership. Science, 305, $782-783$.

Botvinick, M., \& Cohen, J. (1998). Rubber hands 'feel' touch that eyes see. Nature, 391 756.

Burrack, A., \& Brugger, P. (2005). Individual differences in susceptibility to experimentally induced phantom sensations. Body Image, 2, 307-313.

Charles, E. P. (2005). The correction for attenuation due to measurement error: Clarifying concepts and creating confidence sets. Psychological Methods, 10, 206-226.

de Vignemont, F., Tsakiris, M., \& Haggard, P. (2006). Body mereology. In G. Knoblich, I M. Thornton, M. Grosjean, \& M. Shiffrar (Eds.), Human body perception from the inside out (pp. 147-170). New York: Oxford University Press.

Ehrsson, H. H., Spence, C., \& Passingham, R. E. (2004). That's my hand! Activity in premotor cortex reflects feeling of ownership of a limb. Science, 305, 875-877.

Folegatti, A., Farnè, A., Salemme, R., \& de Vignemont, F. (2012). The rubber hand illusion: Two's a company, but three's a crowd. Consciousness and Cognition, 21, 799-812.

Gorsuch, R. L. (1997). Exploratory factor analysis: Its role in item analysis. Journal of Personality Assessment, 68, 532-560.

Haans, A., \& IJsselsteijn, W. A. (in preparation). Individual and situational determinants of rubber-hand illusion: Body image instability, mental motor ability, and fake hand orientation. Manuscript.

Haans, A., IJsselsteijn, W. A., \& de Kort, Y. A. W. (2008). The effect of similarities in skin texture and hand shape on perceived ownership of a fake limb. Body Image, 5 389-394.

Holmes, N. P., Snijders, H. J., \& Spence, C. (2006). Reaching with alien limbs: Visual exposure to prosthetic hands in a mirror biases proprioception without accompanying illusions of ownership. Perception \& Psychophysics, 68, 685-701.

Holmes, N. P., \& Spence, C. (2007). Dissociating body image and body schema with rubber hands. The Behavioral and Brain Sciences, 30, 211-212.

IJsselsteijn, W. A., de Kort, Y. A. W., \& Haans, A. (2006). Is this my hand I see before me? The rubber hand illusion in reality, virtual reality, and mixed reality. Presence: Teleoperators and Virtual Environments, 15, 455-464.

Juhel, J., \& Neiger, H. (1993). The illusory movement sensation paradigm: Some personality correlates. Personality and Individual Differences, 14, 293-296.

Kammers, M. P. M., Verhagen, L., Dijkerman, H. C., Hogendoorn, H., de Vignemont, F., \& Schutter, D. J. L. G. (2009). Is this hand for real? Attenuation of the rubber hand illusion by transcranial magnetic stimulation over the inferior parietal lobule. Journal of Cognitive Neuroscience, 21, 1311-1320. 
Lenggenhager, B., Tadi, T., Metzinger, T., \& Blanke, O. (2007). Video ergo sum: Manipulating bodily self-consciousness. Science, 317, 1096-1099.

Lewis, E., \& Lloyd, D. M. (2010). Embodied experience: A first-person investigation of the rubber hand illusion. Phenomenology and the Cognitive Sciences, 9, 317-339.

Linacre, J. M. (1998). Detecting multidimensionality: Which residual data-type works best? Journal of Outcome Measurement, 2, 266-283.

Linacre, J. M. (2002). Construction of measures from many-facet data. Journal of Applied Measurement, 3, 486-512.

Linacre, J. M. (2003). Data variance: Explained, modeled and empirical. Rasch Measure ment Transactions, 17, 942-943.

Linacre, J. M. (2010). Facets (version 3.67.1). (Computer software). Beaverton, Oregon: Winsteps.com.

Linacre, J. M. (2009). Dichotomizing rating scales. Rasch Measurement Transactions, 23, 1228 .

Longo, M. R., Schüür, F., Kammers, M. P. M., Tsakiris, M., \& Haggard, P. (2008). What is embodiment? A psychometric approach. Cognition, 107, 978-998.

Longo, M. R., Schüür, F., Kammers, M. P. M., Tsakiris, M., \& Haggard, P. (2009). Self awareness and the body image. Acta Psychologica, 132, 166-172.

MacLachlan, M., Desmond, D., \& Horgan, O. (2003). Psychological correlates of illusory body experiences. Journal of Rehabilitation Research and Development, 40, 59-66.

Makin, T. R., Holmes, N. P., \& Ehrsson, H. H. (2008). On the other hand: Dummy hands and peripersonal space. Behavioral Brain Research, 191, 1-10.
Mussap, A. J., \& Salton, N. (2006). A 'rubber-hand' illusion reveals a relationship between perceptual body image and unhealthy body change. Journal of Health Psychology, 11, 627-639.

Paladino, M. P., Mazzurega, M., Pavani, F., \& Schubert, T. (2010). Synchronous multisensory stimulation blurs self-other boundaries. Psychological Science, 21, 1202-1207.

Peled, A., Ritsner, M., Hirschmann, S., Geva, A. B., \& Modai, I. (2000). Touch feel illusion in schizophrenic patients. Biological Psychiatry, 48, 1105-1108.

Rohde, M. Di Luca, M. \& Ernst, M. O. (2011). The rubber hand illusion: Feeling of ownership and proprioceptive drift do not go hand in hand. PloS One, 6 (6 Serial No. e21659).

Schumacker, R. E., \& Lunz, M. E. (1997). Interpreting the chi-square statistics reported in the many-faceted Rasch model. Journal of Outcome Measurement, 1, 239-257.

Shimada, S., Fukuda, K., \& Hiraki, K. (2009). Rubber hand illusion under delayed visual feedback. PloS One, 4 ( 7 Serial No. e6185).

Tsakiris, M., \& Haggard, P. (2005). The rubber hand illusion revisited: Visuotactile integration and self-attribution. Journal of Experimental Psychology. Human Perception and Performance, 31, 80-91.

van Strien, J. W. (1992). Classificatie van links- en rechtshandige proefpersonen [Classification of left- and right-handed participants]. Nederlands Tijdschrift voor de Psychologie en Haar Grensgebieden, 47, 88-92.

Wright, B. D., \& Linacre, J. M. (1994). Reasonable mean-square fit values. Rasch Measurement Transactions, 8, 370. 